\title{
All-optical switching and multistability in photonic structures with liquid crystal defects
}

\author{
Andrey E. Miroshnichenko, ${ }^{1, a)}$ Etienne Brasselet, ${ }^{2}$ and Yuri S. Kivshar ${ }^{1}$ \\ ${ }^{1}$ Nonlinear Physics Center and Center for Ultra-high bandwidth Devices for Optical Systems (CUDOS), \\ Research School of Physical Sciences and Engineering, Australian National University, \\ Canberra Australian Capital Territory 0200, Australia \\ ${ }^{2}$ Centre de Physique Optique Moléculaire et Hertzienne, Université Bordeaux 1, CNRS, \\ 351 Cours de la Libération, 33405 Talence Cedex, France
}

(Received 30 April 2008; accepted 1 June 2008; published online 26 June 2008)

\begin{abstract}
We demonstrate that one-dimensional photonic crystals with pure nematic liquid-crystal defects can operate as all-optical switching devices based on optical orientational nonlinearities of liquid crystals. We show that such a periodic structure is responsible for a modulated threshold of the optical Fréedericksz transition in the spectral domain, and this leads to all-optical switching and light-induced multistability. This effect has no quasistatics electric field analog, and it results from nonlinear coupling between light and a defect mode. (c) 2008 American Institute of Physics. [DOI: 10.1063/1.2949076]
\end{abstract}

The concept of photonic crystals ${ }^{1}$ proposed two decades ago ${ }^{2,3}$ brought a new paradigm to achieve light propagation control in dielectric media. In such periodic photonic structures tuning may be achieved by using materials which are sensitive to external fields, including temperature, electric field, or light itself. In this context, nonlinear photonic crystals have retained much attention due to possible enhancement of nonlinear effects. ${ }^{4}$ Among various nonlinear optical materials that can be implemented in actual photonic crystal devices, liquid crystals (LCs) have been recognized as an attractive alternative material ${ }^{5}$ due to their unique sensitivity to external fields. Since then, many tunable photonic crystal devices based on LC tunability have been suggested and implemented either using complete or partial LC infiltration into the periodic dielectric structure. In the first case, the photonic bandgap is tuned due to refractive index changes of the global structure, ${ }^{6}$ while in the second case a LCinfiltrated layer or hole generates defect modes whose frequencies are controlled by local refractive index changes of LC. ${ }^{7}$ The case of complete infiltration is the most studied one, and it was the first to be demonstrated; it concerns thermal $^{6}$ and electrical ${ }^{8}$ tunability infiltrated one-, two- and three-dimensional photonic structures with LCs.

There exist much less studies concerning optical tuning. One can mention the demonstration using photonic LC fibers, ${ }^{9,10}$ one-dimensional ${ }^{11}$ or planar $^{12,13}$ photonic crystals using absorbing or dye-doped LCs. In these works the resonant interaction of light induces a change of the order parameter, phase transition or surface-mediated bulk realignment. In contrast the nonresonant case, where well-known orientational optical nonlinearity of LC takes place, ${ }^{14}$ has only been explored recently. ${ }^{15}$

In this Letter we address a general problem of all-optical switching in periodic structures with nematic LC (NLCs), based on the optical Fréedericksz transition (OFT). First, we demonstrate a nonlinear feedback due to coupling between light and a defect mode via optical orientational nonlinearities. This feedback could be positive, resulting in all-optical

\footnotetext{
a) Author to whom correspondence should be addressed. Electronic mail:
} aem124@rsphysse.anu.edu.au. multistable switching, or negative, depending on the wavelength detuning from a defect mode. Second, we demonstrate that this coupling leads to different types (first- or secondorder) OFT in periodic structures for the same NLC material. While all-optical bistable switches based on photonic crystals are mainly restricted to inorganic devices, ${ }^{16}$ our results could be envisaged as a promising opportunity for LCinfiltrated photonic crystals technology.

The structure studied in this letter is made of ten bilayers of $\mathrm{SiO}_{2}$ and $\mathrm{TiO}_{2}$ on each side of a NLC layer having a homeotropic alignment (molecules are perpendicular to substrates), as shown in Fig. 1(a). We choose the commercially available E7 LC which is nematic at room temperature and exhibits a second-order OFT for a single slab under linearly polarized excitation. The thicknesses of $\mathrm{NLC}, \mathrm{TiO}_{2}$, and $\mathrm{SiO}_{2}$ layers are $5 \mu \mathrm{m}, 134$ and $143 \mathrm{~nm}$, respectively. We used the dispersion law $n_{\perp}=1.4998+0.0067 / \lambda^{2}+0.0004 / \lambda^{4}$ and $n_{\|}=1.6993+0.0085 / \lambda^{\frac{1}{2}}+0.0027 / \lambda^{4}$ (Ref. 17) for NLC, $n_{\mathrm{SiO}_{2}} \simeq 1.4322+0.0224 /(\lambda+0.28)+0.4005 /(\lambda+0.28)^{2} \quad$ and $n_{\mathrm{TiO}_{2}} \simeq 2.3147-0.2577 /(\lambda+0.11)+202.4 /(\lambda+0.11)^{2}$, respectively, where wavelength $\lambda$ has to be taken in microns. The resulting structure has a bandgap centered around $530 \mathrm{~nm}$ that supports four defect modes, as shown in Fig. 1(b) where the transmission spectra of the unreoriented structure is presented.

The optical properties of the proposed structure are considered to be within the plane wave approximation, so that the system depends only on coordinate $z$ [Fig. 1(a)] and time $t$. The problem is conveniently solved by using the Berreman $4 \times 4$ matrix approach ${ }^{18}$ where Maxwell's equations can be expressed as $\partial \Psi / \partial z=i k_{0} \mathbf{D} \Psi$, where $k_{0}=2 \pi / \lambda$ is the wave vector in vacuum, $\mathbf{D}$ is the Berreman matrix that depends on dielectric permittivity tensor $\epsilon_{i j}$, and $\boldsymbol{\Psi}=\left(E_{x}, H_{y}, E_{y},-H_{x}\right)^{T}$. ${ }^{18}$ The $E_{z}$ component of the light field is obtained from the constitutive equation $\partial_{i} \epsilon_{i j} E_{j}=0$. The whole structure is divided into many layers with constant permittivity, which are described by constant matrices $\mathbf{D}_{n}$. While the thickness of the dielectric layers corresponds to thickness of actual materials, the LC layer is discretized into as many sublayers as necessary to ensure prescribed small variations from one sublayer to another. The resulting matrix thus writes $\mathbf{D}=\Pi \mathbf{D}_{n}$. We ob- 
(a)

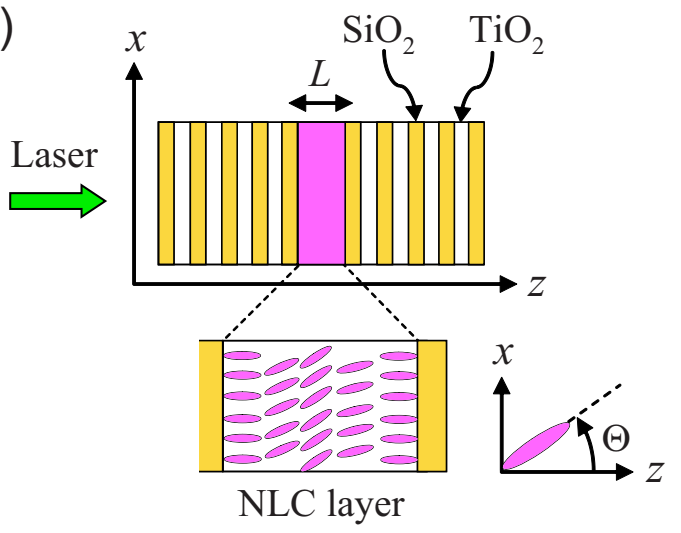

(b)

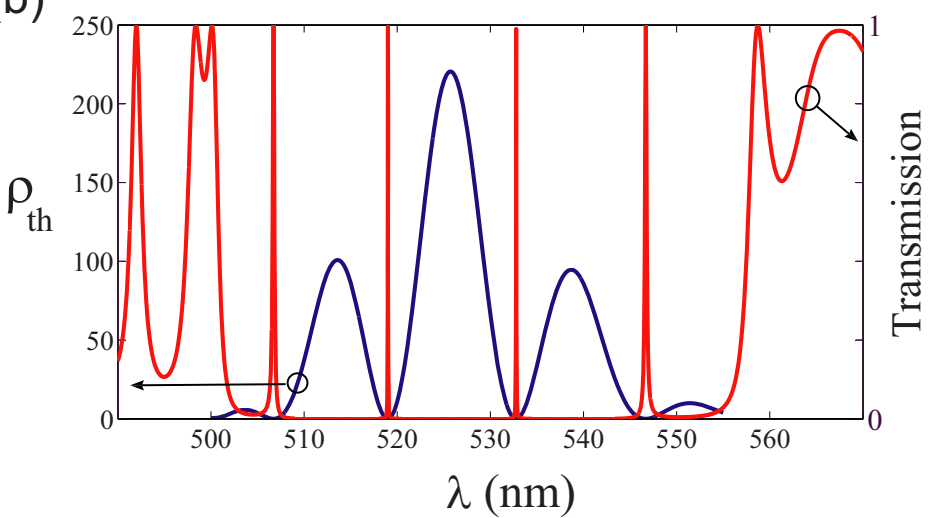

FIG. 1. (Color online) (a) Sketch of the multilayers periodic structure with an embedded NLC defect. (b) Transmission spectra of the unperturbed system (red) and normalized OFT threshold (blue).

tain a light propagation problem for incident $(i)$, transmitted $(t)$, and reflected $(r)$ amplitudes that writes $\boldsymbol{\Psi}^{t}=\mathbf{D}\left(\boldsymbol{\Psi}^{i}+\boldsymbol{\Psi}^{r}\right)$. Inside the NLC layer, the light field is coupled to the EulerLagrange equations that govern the dynamics of the director $\mathbf{n}(z, t)$ and account for dissipative, elastic, and electromagnetic contributions. ${ }^{14}$

We further consider the propagation of light linearly polarized along $x$-axis and assume the director to be constrained in the $(x, z)$ plane, which can be represented by the reorientation angle $\Theta(z, t)$ [Fig. 1(a)]. The problem is solved numerically via the modal expansion procedure detailed in Ref. 19 by searching the reorientation profile as $\Theta(z, t)$ $=\sum_{n=1}^{N} \Theta_{n}(t) \sin (\pi z / L)$, where $N=10$ is enough to ensure accurate results. The needed Frank elastic constants for the NLC are $K_{1}=11.1 \times 10^{-12} \mathrm{~N}$ and $K_{3}=16.0 \times 10^{-12} \mathrm{~N}$. We introduce the normalized intensity $\rho=\left|E_{x}^{i}\right|^{2} / I_{\text {lin }}^{0}$, where $I_{\text {lin }}^{0}$ is the OFT threshold for a single NLC slab and linearly polarized plane wave that corresponds to $\simeq 595 \mathrm{~kW} / \mathrm{cm}^{2}$, and time $\tau=t / \tau_{\mathrm{NLC}}$, where $\tau_{\mathrm{NLC}}$ is the typical relaxation time, ${ }^{14} \tau_{\mathrm{NLC}}$ $\simeq 25 \mathrm{~ms}$ in our case. Note that the OFT threshold value could be reduced by up to two order of magnitude using a small addition of dye into the NLC. ${ }^{20}$ We define the total light-induced phase delay in the presence of reorientation, $\Delta(\tau)=k_{0} \int_{0}^{L}\left[n_{e}(z, \tau)-n_{o}\right] d z$, with $n_{o / e}$ being the ordinary and effective extraordinary refractive indexes.

First, we calculate the OFT threshold $\rho_{\text {th }}$ above which the NLC is reoriented for pump wavelength $\lambda_{p}$ lying inside the bandgap [see Fig. 1(b)]. As was shown in Ref. 15, the reorientation threshold is drastically reduced at a defect wavelength with respect to the single NLC slab $\rho_{\text {th }}\left(\lambda_{d}\right) \ll 1$, caused by a very strong light confinement at the defect mode placed in a periodic structure. A reduction factor up to $10^{3}$ is obtained for the present structure. In contrast, a significant increase of the threshold is observed when $\lambda_{p}$ is strongly detuned from defect mode frequencies [Fig. 1(b)]. Indeed, the smaller the detuning parameter $\delta=\lambda_{p}-\lambda_{d}$ is, the better light confinement inside the defect NLC layer, which leads to the lower reorientation threshold.

Although the OFT threshold is independent of the sign of $\delta$ for $\lambda_{p} \approx \lambda_{d}$ (local parabolic approximation), the type of the light-induced reorientation strongly depends on it. This point is illustrated in Fig. 2(a) where the reorientation diagram $\Delta$ as a function of $\rho$ is shown for $\delta= \pm 0.25 \mathrm{~nm}$ around $\lambda_{d}=532.75 \mathrm{~nm}$. A negative detuning leads to a second-order OFT, as it is the case for the NLC slab alone, whereas a positive detuning exhibits a first-order OFT with an relative hysteresis width of almost $100 \%$. To understand this behavior we note that defect mode frequencies underwent a redshift proportional to the increase of the averaged refractive index inside the NLC defect layer due to molecular reorientation. Consequently, recalling that the threshold intensity has a parabolic profile around defect modes $\rho_{\mathrm{th}}\left(\lambda_{p}\right)-\rho_{\mathrm{th}}\left(\lambda_{d}\right)$ $\propto\left(\lambda_{p}-\lambda_{d}\right)^{2}$, negative detuning $\delta<0$ leads to a negative feedback and positive detuning $\delta>0$ is accompanied by a posi-
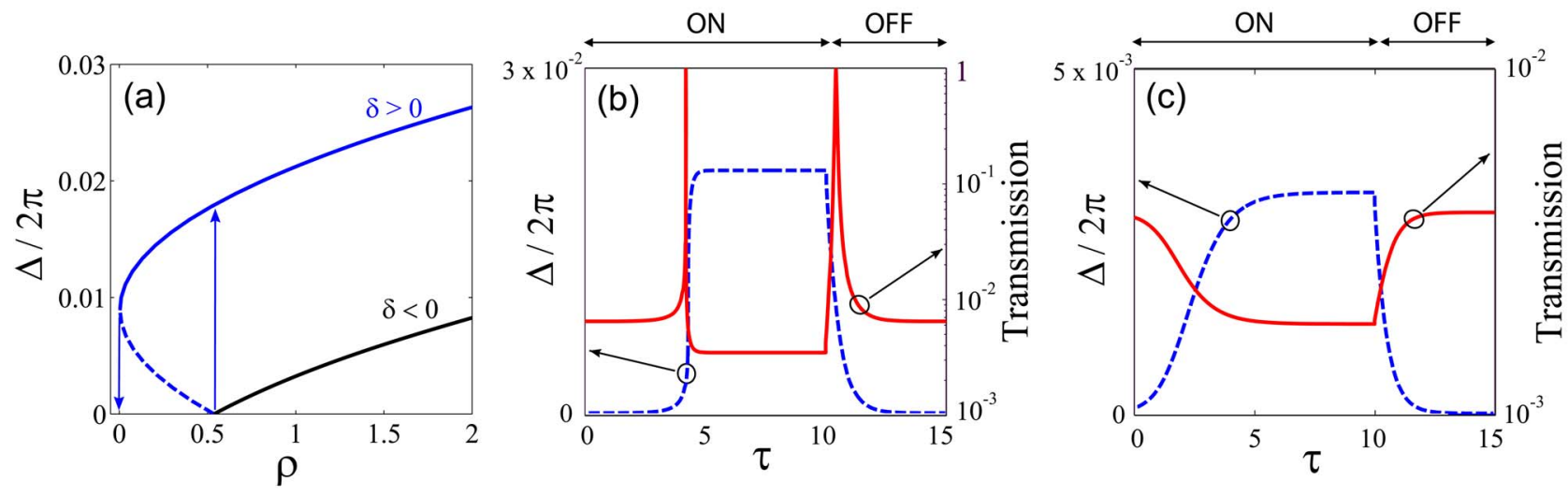

FIG. 2. (Color online) (a) Reorientation diagram for positive (blue) and negative (black) detuning $\delta= \pm 0.25 \mathrm{~nm}$ around $\lambda_{d}=532.75 \mathrm{~nm}$. Solid (dashed) lines refers to stable (unstable) states. Corresponding dynamics for (b) $\delta>0$ and (c) $\delta<0$ of the phase delay $\Delta$ and pump light transmission for square-shaped temporal excitation with $\rho=1$. 

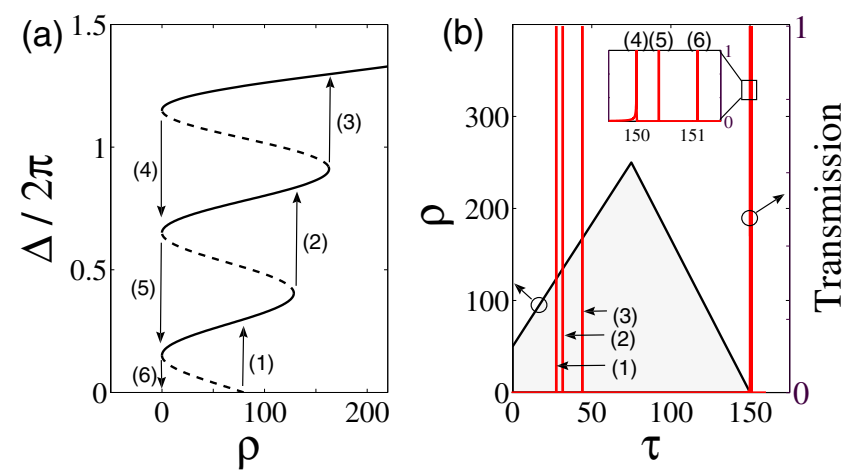

FIG. 3. (Color online) (a) Multistable reorientation diagram for positive detuning at $\lambda_{p}=537 \mathrm{~nm}$. Solid (dashed) lines refers to stable (unstable) states. (b) Corresponding dynamics of pump light transmission for triangleshaped temporal excitation.

tive feedback. All-optical bistable switching is thus achieved by properly chosen pumping wavelength $\lambda_{p}>\lambda_{d}$.

The dynamics of the all-optical switching process is illustrated in Fig. 2(b), where the unperturbed system is excited with $\delta>0$ and $\rho>\rho_{\text {th }}\left(\lambda_{p}\right)$ for $\tau<10$ and $\rho=0$ for $\tau>10$. Two transmission peaks are observed, the first one at excitation stage and the second one during relaxation process. These peaks correspond to a transient resonance between $\lambda_{d}$ and $\lambda_{p}$ due to the displacement of the defect modes inside the bandgap caused by reorientation. The full width at half maximum pulse duration of the first and second peaks are $\simeq 4 \times 10^{-4} \tau_{\mathrm{NLC}}$ and $\simeq 8 \times 10^{-2} \tau_{\mathrm{NLC}}$, namely, $10 \mu$ s and $2 \mathrm{~ms}$ in the present case. Initial conditions at $\tau=0$ are taken as $\left(\Theta_{1}=10^{-2}, \Theta_{2, \ldots, N}=0\right)$, which mimics a thermal orientational fluctuation in the NLC slab. As a result, the smaller the detuning parameter is, the smaller is the required intensity to perform all-optical switching in periodic structures. A compromise has nevertheless to be found to preserve lightinduced phase delay discontinuity since it vanishes for $\delta \rightarrow 0^{+}$.

Multistable all-optical switching can also be achieved in such a structure for larger intensities, caused by larger molecular reorientation resulting in larger changes of the refractive index. This leads to a possibility of many defect modes passing the pumping wavelength $\lambda_{p}$, and, consequently, to a series of reduction of the pumping intensity $\rho$ giving reorientation boosts. An example is shown in Fig. 3(a) for $\lambda_{p}$ $=537 \mathrm{~nm}$. The multistability is evidenced by several coexisting stable states in the reorientation diagram. The corresponding switching dynamics is shown in Fig. 3(b) when a linear ramp of excitation light intensity is used. Each of six peaks of the transmission dynamics is reminiscent of a plateau in the reorientation diagram, as indicated by labels num- bered (1)-(6), and has the same origin as those discussed above.

In conclusions, we have predicted multistable all-optical switching resulting from an unique nonlinear coupling between light and defect modes in a periodic dielectric structure that contain a NLC defect layer. Moreover, depending on the pumping wavelength $\lambda_{p}$ the same NLC material may demonstrate first-or second-order OFT in periodic structures. It is important to note that there is no quasistatics electric field analog to the optical case studied here because the electro-optical tunability explored earlier ${ }^{21}$ is not accompanied by a coupling between excitation field and defect modes. Additional functionalities are expected when nonplanar molecular light-induced reordering takes place. ${ }^{19}$

This work was supported by Discovery and Center of Excellence projects of the Australian Research Council.

${ }^{1}$ See, e.g., J. D. Joannopoulos, R. D. Meade, and J. N. Winn, Photonic Crystals: Molding the Flow of Light (Princeton University Press, Princeton, NY, 1995), and references therein.

${ }^{2}$ E. Yablonovitch, Phys. Rev. Lett. 58, 2059 (1987).

${ }^{3}$ S. John, Phys. Rev. Lett. 58, 2486 (1987).

${ }^{4}$ M. Soljačić and J. D. Joannopoulos, Nat. Mater. 3, 211 (2004).

${ }^{5}$ K. Busch and S. John, Phys. Rev. Lett. 83, 967 (1999).

${ }^{6}$ K. Yoshino, Y. Shimoda, Y. Kawagishi, K. Nakayama, and M. Ozaki, Appl. Phys. Lett. 75, 932 (1999).

${ }^{7}$ Y.-K. Ha, Y.-C. Yang, J.-E. Kim, H. Y. Parka, C.-S. Kee, H. Lim, and J.-C. Lee, Appl. Phys. Lett. 79, 15 (2001).

${ }^{8}$ D. Kang, J. E. Maclennan, N. A. Clark, A. A. Zakhidov, and R. H. Baughman, Phys. Rev. Lett. 86, 4052 (2001).

${ }^{9}$ T. T. Alkeskojld, L. A. Bjarklev, D. S. Hermann, A. Anawati, J. Broeng, J. Li, and S. T. Wu, Opt. Express 12, 5857 (2004).

${ }^{10}$ J. Tuominen, H. J. Hoffrén, and H. Ludvigsen, J. Eur. Opt. Soc. Rapid Publ. 2, 07016 (2007).

${ }^{11}$ H. Yoshida, C. H. Lee, Y. Miura, A. Fujii, and M. Ozaki, Appl. Phys. Lett. 90, 071107 (2007).

${ }^{12}$ B. Maune, J. Witzens, T. Baehr-Jones, M. Kolodrubetz, H. Atwater, A. Scherer, R. Hagen, and Y. Qiu, Opt. Express 13, 4699 (2005).

${ }^{13}$ P. El-Kallassi, R. Ferrini, L. Zuppiroli, N. Le Thomas, R. Houdré, A. Berrier, S. Anand, and A. Talneau, J. Opt. Soc. Am. B 24, 2165 (2007).

${ }^{14}$ N. V. Tabiryan, A. V. Sukhov, and B. Ya. Zel'dovich, Mol. Cryst. Liq. Cryst. 136, 1 (1986).

${ }^{15}$ A. E. Miroshnichenko, I. Pinkevych, and Y. S. Kivshar, Opt. Express 14, 2839 (2006).

${ }^{16}$ M. Notomi, A. Shinya, S. Mitsugi, G. Kira, E. Kuramochi, and T. Tanabe, Opt. Express 13, 2678 (2005).

${ }^{17}$ J. Li, S.-T. Wu, S. Brugioni, R. Meucci and S. Faetti, J. Appl. Phys. 97, 073501 (2005).

${ }^{18}$ D. W. Berreman, J. Opt. Soc. Am. 62, 502 (1972).

${ }^{19}$ E. Brasselet, T. V. Galstian, L. J. Dubé, D. O. Krimer, and L. Kramer, J. Opt. Soc. Am. B 22, 1671 (2005).

${ }^{20}$ L. Marrucci, D. Paparo, P. Maddalena, E. Massera, E. Prudnikova, and E. Santamato, J. Chem. Phys. 107, 9783 (1997).

${ }^{21}$ R. Ozaki, H. Moritake, K. Yoshino, and M. Ozaki, J. Appl. Phys. 101, 033503 (2007) 